\title{
Redrawing Therapeutic Boundaries: Microbiota and Cancer
}

Jonathan Sholl*, Gregory D. Sepich-Poore, Rob Knight, Thomas Pradeu*

\section{Authors' affiliations}

- JS: Univ. Bordeaux, CNRS, ImmunoConcEpT, UMR 5164, 33000 Bordeaux, France

"Correspondence: jonathan.sholl@u-bordeaux.fr (J. Sholl)

- GSP: Department of Bioengineering, University of California San Diego, La Jolla, CA, USA

- RK: Departments of Bioengineering, Pediatrics, and Computer Science and Engineering, and Center for Microbiome Innovation, University of California San Diego, La Jolla, CA, USA

- TP: Univ. Bordeaux, CNRS, ImmunoConcEpT, UMR 5164, 33000 Bordeaux, France

"Correspondence: thomas.pradeu@u-bordeaux.fr (T. Pradeu)

\section{Abstract}

The unexpected roles of the microbiota in cancer challenge explanations of carcinogenesis that focus on tumor-intrinsic properties. Most tumors contain bacteria and viruses, and the host's proximal and distal microbiota influence both cancer incidence and therapeutic responsiveness. Continuing the history of cancer-microbe research, these findings raise a key question: to what extent is the microbiota relevant for clinical oncology? We approach this by critically evaluating three issues: how the microbiota provides a predictive biomarker of cancer growth and therapeutic responsiveness, the microbiota's causal role(s) in cancer development, and how therapeutic manipulations of the microbiota improve patient outcomes in cancer. Clarifying the conceptual and empirical aspects of the cancer-associated microbiota can orient future research and guide its implementation in clinical oncology.

\section{Keywords}

Cancer; microbiota; biomarkers; causality; therapeutic modulation; network medicine 


\section{Introducing the microbiota to oncology}

2 Recent work elucidating the microbiota's various roles in cancer initiation, progression and treatment challenges the predominant view that cancer can be explained primarily in terms of tumor-intrinsic properties [I]. Not only do tumors contain bacteria [2,3], viruses [4] and sometimes fungi [5], but both cancer incidence and response to various cancer therapies are influenced by the host's microbiota [6]. Despite the overall enthusiasm around the microbiota in most areas of current biology and medicine [7-IO], these discoveries came as a surprise because they expanded the scope of what was considered relevant for oncology. This research has also been met with skepticism; amidst the controversial history of using microbes to explain or treat cancer, it has often been difficult to establish the reproducibility, efficacy and safety of these approaches [II]. Moreover, in certain cancer types, the relative importance of microbial versus tumor cell mechanisms and their interactions in carcinogenesis remains debated [I2]. It is thus still an open question to what extent the microbiota is relevant for clinical oncology [13-16], and whether the role of the microbiota in cancer requires an explanatory shift beyond tumor-intrinsic features.

After providing some brief historical background on the use of microbes/infections in cancer research and therapy, we illustrate how data on cancer-associated microbiota question several tightly held assumptions in oncology [I7-I9]. We then evaluate the relevance of the microbiota for oncology by clarifying three complementary perspectives. First, can the microbiota be used as a biomarker of cancer growth and therapeutic responsiveness? Second, can the microbiota be causally linked to cancer development? Finally, can the microbiota be therapeutically manipulated to improve the treatment and course of cancer, and if so, how? By clarifying several conceptual and empirical challenges at the intersection of oncology and microbiology, this conceptual review proposes the oncological utility of cancer-associated microbiota in patient diagnosis, prognosis, and treatment.

\section{Historical background: from microbes to mutations}

There is a long tradition of using microbes-particularly bacteria and viruses-in cancer therapy. In a broad sense, this may date back to observations in ancient Egypt and Greece that tumor regression followed infections and/or fevers [II,20,2I]. The first scientific attempts at modulating the immune system to treat cancer appear to emerge in the late $19^{\text {th }}$ Century with the German physicians Wilhelm Busch and Friedrich Fehleisen who independently noticed tumor regression in several patients following erysipelas infections caused by 
1 Streptococcus pyogenes. In the early $20^{\text {th }}$ Century, this tradition was advanced by one of the

2 'fathers' of immunotherapy, William Coley, who, after tracking down a patient in New York 3 who experienced spontaneous regression of an egg-sized sarcoma following erysipelas, started controversially injecting live and later heat-inactivated bacteria ("Coley's toxins") into his patients with inoperable cancer [2I]. Despite the apparent success of Coley's method to achieve $\sim 30 \%$ long-term remission in II types of malignancies across $\sim 2$ IO patients before I940 [22], his findings and methods were not well received by the oncology community due to poorly understood mechanisms, painful fevers (often associated with therapeutic responses), difficult reproducibility, and non-trivial risks of death in immunocompromised patients. Some suggest that as surgery and radiotherapy advanced, and antibiotics and antipyretics were commonly used to enhance hygiene or suppress undesirable immune responses, this sterilizing environment may have obscured the potential anti-tumoral roles for microbes and immune or febrile reactions [2I].

Throughout the $2 \mathrm{O}^{\text {th }}$ Century, there was a parallel tradition of considering whether microbes play a causal role in carcinogenesis and progression [23]. Amidst interest for cancer vaccines, first Thomas Glover and later Virginia Livingston-Wheeler argued that bacteria could be cultivated from tumors and that cancer had a bacterial origin [24,25]. However, Glover's findings were not reproducible by researchers at the National Institutes of Health and Livingston-Wheeler's research was criticized for not controlling for contamination [24]. As this bacterial research was being abandoned (though not entirely [26]), Peyton Rous made the tentative observation in I9II that specific 'agents' from a chicken tumor could be transmitted to healthy chickens, thereby reproducing an avian tumor resembling human neoplasms [27]. Initially met with outright rejection or considerable skepticism [28], Rous's findings were eventually vindicated, opening the field of tumor virology [23] — canonized by his receipt of the Nobel Prize in 1966. While a variety of viruses have been linked to several cancers, such as Epstein-Barr, hepatitis viruses, and human papilloma-the most recent being the Merkel cell polyomavirus described in 2008 - the viral origins of cancer proved limited and ultimately gave way to a focus on internal etiologies such as cellular mechanisms and mutations. Notably, the 1989 Nobel Prize to Michael Bishop and Harold Varmus signaled this major shift in thinking, for they found that many retroviral oncogenes, including from Rous's sarcoma virus, had a cellular (non-viral) origin and were found in many animal species [29]. As the presumed origin of cancer moved from external to internal factors, it engendered research into cancer genomics. 
Together, these historical traditions exhibit the persistent challenges, and even

2 reluctance, for evaluating the importance of microbes in oncology. One interesting tension 3 running through this history concerns the relative importance of microbes for conceptualizing tumorigenesis: is cancer formation and progression primarily intrinsic or extrinsic [28]? We have seen that throughout the $20^{\text {th }}$ Century microbes were viewed as an extrinsic factor that might have a direct causal or therapeutic role, but paradoxically, this perspective eventually catalyzed the study of cancer genetics [30] and the focus on cellintrinsic mechanisms (while clearly acknowledging extrinsic triggers).

Recent research is not only examining specific microbes but also the microbiota, or communities of microbes that inhabit and influence the human body and their potential role in cancer progression and treatment [I8]. The microbiota colonizes tumors and even individual tumor cells [18], prompting us to once again ask, for instance, in what sense the microbiota is an extrinsic or intrinsic causal and therapeutic factor. The additional findings that patient responsiveness to cancer therapies (e.g., immune checkpoint inhibitors (ICI) or chemotherapies) depends on the microbiota in the host appear to blur the intrinsic-extrinsic distinction [I4,3I,32]. As advances continue to uncover complex interactions among the microbiota, cancer cells, and the host's immune responses [33], the microbiota have become a manipulable tool at once external to and part of the host and its tumor(s). As such, the microbiota is no longer an accidental environmental factor to be suppressed but appears necessary for understanding and effectively treating cancer.

\section{The microbiota provides distinct biomarkers in oncology}

A key area in oncology comprises the search for cancer-specific biomarkers that enable accurate predictions about patient diagnosis, prognosis, and treatment. Going beyond tumor-intrinsic factors, many have begun investigating whether the microbiota, and its functions or metabolites, can alone serve as non-human biomarkers for cancer [34-36]. Here, we discuss how the microbiota constitute distinct types of biomarkers [37].

First, blood-derived microbial DNA and specific alterations in localized microbial communities have recently been put forth as possible diagnostic biomarkers for various cancers [2,3,38], though their real-world performance remains to be seen [I8]. As prognostic and risk biomarkers for evaluating cancer progression, patterns in microbial communities in patients may help explain inter-individual variation in which mutations become cancers [39], personal risk of acquiring cancer [40], and the anti- or pro-tumor function of specific 
1 specific to a given cancer type (the 'intratumoral microbiome'), but also the host microbiota

2 at distant sites. For instance, alpha diversity in the intratumoral microbiota is predictive of

3 short versus long-term survival in pancreatic cancer [15], patterns of microbial community changes in lung tissues may be markers of lung cancer progression [4I], and gut microbiota composition can inform the risk of developing colorectal cancer [42]. Distally, gut microbiota can help assess the risk of progression from liver disease to cancer [43] and determine patients at risk of tumor metastasis in breast cancer [35], and periodontal microbes appear to stratify risk for developing pancreatic cancer [44].

Next, as therapeutic response biomarkers, the microbiota can surprisingly determine a patient's likelihood of responding to treatment [45]. Bourgeoning evidence suggests how clinical antibiotics is a predictor of poor survival in various cancers (with some exceptions [I4,46]) and might undermine cancer therapies [47]. Conversely, increasing attention is given to the ability of the microbiota to support the efficacy of immune checkpoint inhibitors $[48,49]$, as well as traditional chemotherapies [14,50]. Researchers have extracted specific microbial species from the feces of responders and non-responders and have shown how these phenotypes are replicated in mice or humans upon receipt of concomitant microbes $[6,51,52]$. These data provide persuasive examples of how the microbiota is linked to therapeutic efficacy and in some cases may outperform traditional biomarkers such as tumor mutational load [3I]. Some researchers also suggest that the functional traits they exhibit could have more explanatory value than the taxa compositions alone, although this remains to be rigorously tested [53].

Ultimately, predictions about cancer risks and progression are not solely tied to tumor-intrinsic properties but are coupled with, and in some cases superseded by, local and distant microbial signatures $[4 \mathrm{I}, 43]$. As such, the microbiota may eventually allow for better diagnoses and appears crucial for predicting the health and survival of the cancer-bearing host, highlighting its relevance for oncology. However, the transition from correlative signatures and biomarkers to causative factors raises challenges since many of these microbial biomarkers comprise entire communities of organisms in comparison to individually assayed host biomarkers [54].

\section{Establishing causality in cancer-microbiota interactions}

Although experts agree that a handful of microbes have causal roles in carcinogenesis [55], many complexities exist, including how particular microbes and communities can aid in tumorigenesis without being direct causal agents, or can even protect against tumorigenesis 
1 [I8]. Determining causality requires a careful analysis of the relevant context and the distinct

2 roles that microbes play under various physiological conditions. Box I addresses some of the

3 mechanisms identified for the roles discussed below and Figure I offers a visual representation.

\section{Pathogenic microbes in cancer}

From the perspective of microbes as pathogens - generally considered extrinsic causes - it is well accepted that key bacteria and viruses have oncogenic effects in humans. There are currently II agents recognized as bona fide 'oncomicrobes' in humans (IARC Working Group 2012), including Helicobacter pylori, human papillomavirus (HPV), hepatitis B virus (HBV), and hepatitis $\mathrm{C}$ virus (HCV), Epstein-Barr, herpesviruses, and various polyomaviruses [57]. Moreover, several seemingly pro-carcinogenic bacteria, such as Bacteroides fragilis, Enterococcus faecalis, Fusobacterium and pks+ strains of Escherichia coli, all appear to have carcinogenic capacities through their effects on the host immune system, mutagenesis, and inflammation $[48,58,59]$.

However, even where specific microbes are shown to have cell-transforming abilities, they often occur alongside inflammation or specific changes to the microbial milieu, such that "causal" microbes may be necessary yet insufficient for tumorigenesis and progression $[58,60]$. Thus, while causal links exist, the influence of the broader microbial community and the physiological responses of the host should also be considered.

\section{Microbes living in and traveling with the tumor}

Recent data supports the perspective that microbes pervasively colonize tumors-evidence that falsifies the assumption of cancer's sterility [2-5,6I], a theory possibly kept alive by sterility assumptions of various organs, such as the lungs or bladder [62]. In fact, distinct cancers have cancer type-specific microbial signatures [2,3]: a microbiome unique to each cancer with varying ratios of, e.g., Proteobacteria and Firmicutes, appearing in higher loads in tumors than in adjacent normal tissue. It is perhaps not surprising to find microbes in gastrointestinal cancers, which are anatomically proximal to the gut microbiota, but intratumoral microbiomes have also been found in tissues distal from the gut, such as in breast, lung, ovary, melanoma, bone, and brain tumors. In these studies, many bacteria detected in tumors appear to be live, cell-wall deficient bacteria, which are exclusively intracellular bacteria and are mainly found in cancer cells and immune cells [2]. It remains unknown how many of these bacteria are merely passengers rather than active participants in a nutrient-rich and immunosuppressed environment. 
Additionally, the microbiota can be seen as migrators. For instance, microbes found

2 in primary colorectal tumors have also been found in matched metastatic liver tumors [6I].

3 Although originally thought to hitchhike within metastatic cancer cells [6I], new evidence suggests that colorectal cancer bacteria may actually travel to the liver ahead of metastatic cells and prepare a pro-tumoral, pro-inflammatory environment for them to later seed [63]. These metastatic processes remain uncharacterized in most cancer types.

While specific taxa were identified in these studies, their functional repertoire and spatial distribution within tumors remain poorly characterized, obscuring causal roles they may play in cancer progression [53]. Determining the pathogenicity of these tumoral colonizers is complicated by the observation that, in some cases, the composition of specific microbes in tumors may improve patient outcomes [I5] or enhance immunotherapy response [2].

\section{The role of the resident microbiota in promoting or inhibiting cancer}

Another perspective studies the microbiota as an oncological regulator. This idea is supported by research showing how the resident microbiota can play a dual role in promoting or inhibiting cancers.

There are multiple ways in which the microbiota promotes cancer growth and progression. Bacterial infections trigger inflammation and innate immunity pathways, which in turn create a tumor-promoting microenvironment [63-65]. A second pathway is through dysbiosis, a widely discussed and sometimes contested concept, generally referring either to a loss of beneficial microbes, an expansion of pathobionts, or reduced diversity [6668]. While in some cases it is unclear whether dysbiosis is driving inflammation and thus tumorigenesis, or whether inflammation drives the dysbiosis, there are nevertheless strong links between the microbial community changes, inflammation, and tumor promotion [58]. Furthermore, when barrier epithelial cells are damaged (an innate immune defense), the "normal" resident microbiota can further damage these cells or underlying tissues, induce genetic instability via DNA-damaging reactive oxygen and nitrogen species [48], or translocate into circulation causing systemic inflammatory responses [69].

While certain microbes are pathogenic, others support the body's antitumoral responses. The microbiota can epigenetically prime myeloid cells, such as dendritic cells and macrophages, for optimal responsiveness to tumors [65], an effect that is significantly reduced in germ-free mice models. Several bacterial species have also been associated with anticancer immunosurveillance [70], with specific intratumoral microbial compositions linked to better chances of survival [15] or response to immunotherapy [2]. It remains 
undetermined whether the diversity of gut microbes facilitating positive outcomes can be explained by shared functional output of specific metabolites (e.g., short-chain fatty acids) that contribute to reducing inflammation and/or ensuring intestinal barrier integrity [71,72].

\section{Box I: Microbiota-Cancer Causal Mechanisms}

When viewed as cancer pathogens, we can distinguish direct and indirect mechanisms:

- Direct pathogenic mechanisms [18,73-75]: genotoxic or cytotoxic mutagenesis (colibactin, cytolethal distending toxins) via DNA alkylation, DNAse activity, and ROS/NOS production; activating $\beta$-catenin or $\mathrm{PI} 3 \mathrm{~K} / \mathrm{AKT}$ pathways.

- Indirect pathogenic mechanisms [48,65,72,76,77]: following translocation, microbiota promote inflammatory $\gamma \delta$ T cells, DNA damaging ROS-producing neutrophils; their metabolites can hinder immunosurveillance of human NK and T cell activity; following mucosal damage in gut, microbes regulate cytokines (e.g., IL-6, II, I8, 22).

As tumoral colonizers and migrators, there are various potential mechanisms:

- Intratumoral TME-colonizing mechanisms [I5,I8,46,65,78]: producing genotoxins, Tcell mediated inflammation, suppressing local antitumor immunity (short-chain fatty acids (SCFAs) inducing Treg production), enzymatically aiding chemoresistance (bacterial cytidine deaminase degrading gemcitabine), activating the host's MBL-C3 axis.

- Mechanisms of migrators [6I,63]: potentially intracellular migration with metastatic cancer cells; bacteria such as E. coli can open the gut vascular barrier and thereby translocate to the liver where they recruit immune cells (macrophages and inflammatory monocytes) and aid in the maturation of a premetastatic niche.

When viewed as cancer regulators, we find mechanisms for promoting and inhibiting tumorigenesis:

- Promoter mechanisms [18,33,48,65,77,78]: contributing to a pro-inflammatory microenvironment, stimulating IL-I and IL23 from myeloid cells or IL-I7 from ThI7 cells; tumor-promoting pathogens escaping immune control (dysbiosis); activating inflammasomes $\left(\mathrm{NLRP}_{3}, 6\right)$ via SCFAs, in turn increasing tumor promoting IL-22 (positive feedback loop); promoting metastasis by upregulating tumor matrix metalloproteinases.

- Inhibiting mechanisms [79-83]: The microbes growing in and around tumors can inhibit growth through the production of anti-inflammatory metabolites, particularly SCFAs such as butyrate and propionate, which have been shown to affect gene expression, cell proliferation and cell death; mimicry between microbial or phage antigens and cancer antigens thereby causing antitumor immune response. 


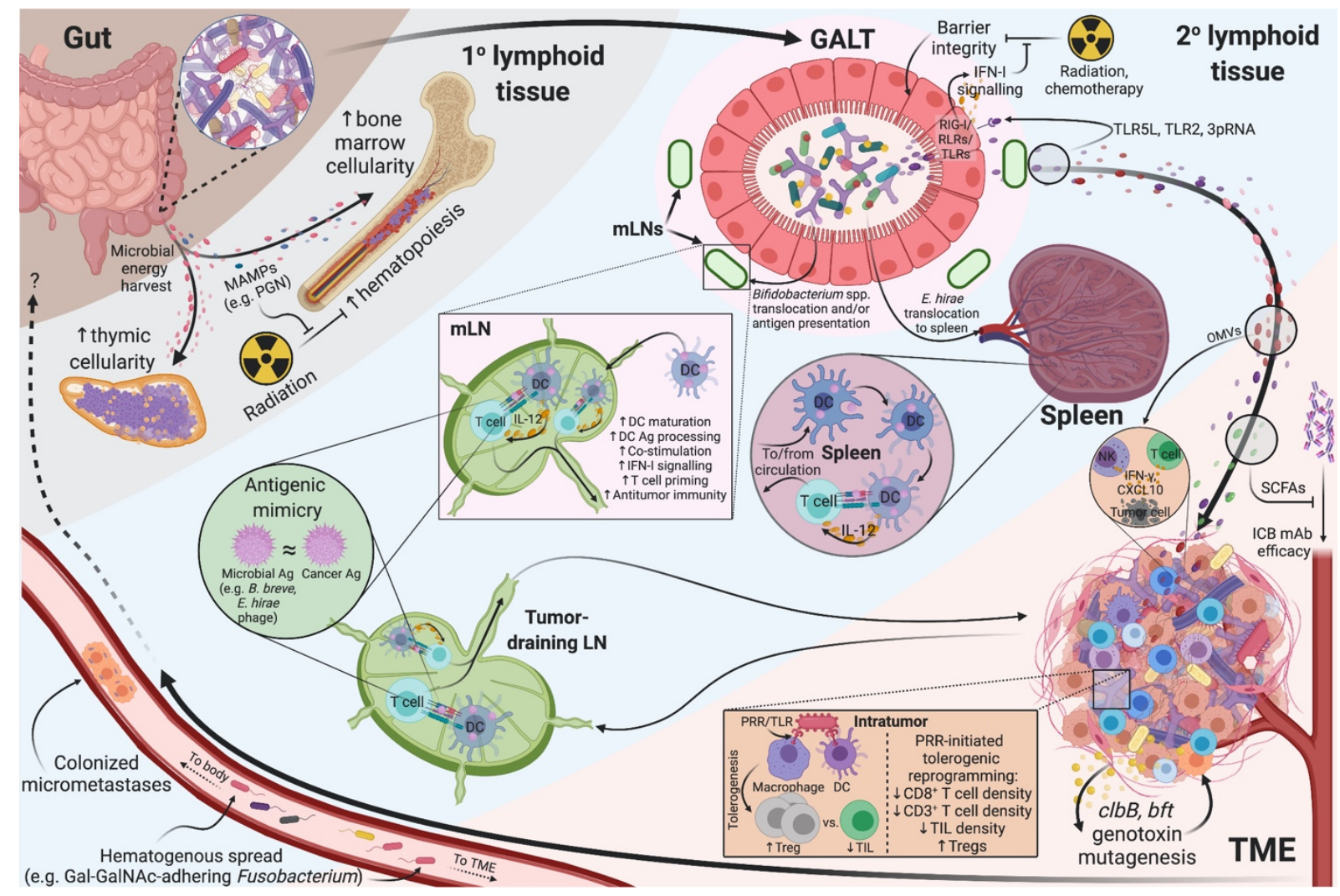

\section{$4 \quad$ Reevaluating causality}

5 Several challenges remain for evaluating causal claims of the cancer-associated microbiota.

6 These include the primary way researchers conclude causality in microbiome studies: rodent models. There are limitations of uneven colonization in recipient animals, the prevention of novel communities forming upon colonization, the lack of ecological factors in these models that were important for producing host disease states, and the difficulty of getting recipient animals to adapt to microbes with which they did not co-evolve [84]. Although some cancer studies have performed human fecal microbiota transplants [5I,52], these are rare. Causal claims are further complicated due to some infections having long latency periods (e.g., human T-cell leukaemia virus type I), the fact that many microbes are widespread and yet their associated cancer is rare, and that causal mechanisms may vary during the time course of carcinogenesis [23].

Moreover, this research faces the immense challenge of specifying causality amidst the complex variations of host-microbe and microbe-microbe dynamics [6o]. Microbes exist within ecosystems, and even if single microbes can be linked to or associated with various cancers, they also tend to be accompanied by shifts in other microbial taxa [72]. This is further complicated when considering that phages preying on bacteria may influence these 
dynamics [83]. Finally, there is the issue of context-dependency [53,70,85], such as whether specific microbes contributing to cancer depends on host physiology (e.g., inflammation).

One proposed way forward is to incorporate ideas from systems medicine or ecology [72,86-92]. For instance, we can track how perturbations in one part of the microbe-host ecology will result in adjustments, compensations, or disruptions to other parts of this system [93]. The challenge is to determine how to accurately define these systems and their relevant causal factors without sacrificing explanatory precision or clinical utility.

\section{Manipulating the microbiota for cancer therapy}

While establishing etiology is central to this research, there is a closely related issue of investigating the effects of host-microbiota interactions on cancer therapies. Evidence that the microbiota or its metabolites modulate, or in some cases enable, the outcomes of cancer therapies is increasingly well-supported [6]. As microbes and the microbiota play the role of therapeutic mediators, this suggests that host physiology is not solely responsible for whether a cancer treatment is effective. Microbes are not just accidental aspects of host physiology that can be ignored or even eradicated, as is commonly done to treat or prevent opportunistic infections [94], possibly obscuring their beneficial roles in cancer [2I]. In fact, they may sometimes be necessary for treating cancer. Set against the above history, the microbiota is perhaps less of a medical 'breakthrough' [95] than an increasingly promising and better understood therapeutic target.

\section{What the microbiota can do to cancer therapies}

While modulating host immune responses to tumors has long been a target of immunotherapies, it was only recently possible to ask whether a patient's microbiota might undergird treatment efficacy. This intriguing role of the microbiota as a mediator has been confirmed, in part, by showing that antibiotic treatments reduce the efficacy of various cancer therapies: in the absence of commensal microbiota, the immune system will either not, or to a lesser degree, be activated by immunotherapies [70,71,96]. Returning to the research on immune checkpoint inhibitors (ICIs), antibiotic-induced gut dysbiosis can inactivate the antitumoral $\mathrm{T}$ cell responses, and re-introduction of several bacterial species, their proteins, and/or their metabolites appears to restore the activity of these therapies [7I,97-99]. Moreover, while gut commensals seem to enhance some ICIs (anti-PD-I or anti- 
which microbiota and/or their metabolites support immunotherapy efficacy remains under

2 investigation [IOI].

The microbiota also appears to mediate conventional cancer therapies. For instance, alterations in gut commensals support the efficacy of total body irradiation (TBI) as a conditioning regime for adoptive T-cell transfer therapy [58], and the beneficial effects of TBI are reduced by antibiotics. Conversely, certain commensals may be important for patient survivability of whole body radiotherapy [IO2], and intestinal fungi appear to modulate antitumoral responses to radiation therapy in mice [I03]. Synergistic antitumor effects seem to be driven by translocating bacteria from the gut into neighboring tissues and subsequently inducing immunostimulation [58], or by priming tumor-infiltrating myeloid cells [65]. Similar antitumor effects have been found with chemotherapies using oxaliplatin or cyclophosphamide: the antitumor immune responses are primed and/or enhanced by commensal bacteria [50,97,I04]. For instance, cyclophosphamide disrupts gut mucosal integrity, thereby inducing the translocation of specific gram-positive bacteria into secondary lymphoid organs, which then stimulate the production of antitumoral Thi7 cells and Thı immune responses.

One key question is whether these responses are due less to the individual species found in the so-called 'responders' than to specific communities, 'consortia', or even the entire ecosystem with which these species are associated [70]. Deconvolving microbemicrobe and host-microbe interactions are necessary to address this in detail.

\section{Redrawing the boundaries of therapeutic intervention}

Using microbes to treat and better understand cancer fits within the longer historical traditions while expanding the current one [17,IO5-IO7]. A nuanced development, though, concerns the shift from using exogeneous microbes (e.g., Coley's treatments) to manipulating endogenous ones to stimulate an antitumor immune response. In this sense, the extrinsicintrinsic distinction concerning the microbiota's relation to cancer is dissolving: the mechanism is at once external and internal, depending on one's perspective.

However, a large problem for redrawing the therapeutic boundary becomes apparent when considering the context-dependency of microbes in carcinogenesis. For instance, while bacteria such as H. pylori and viruses such as herpesvirus and Epstein-Barr can all be carcinogenic, they can also cooperate with commensals and thereby offer some protection against other diseases and infections [48,I08]. Evolutionary trade-offs will thus have to be carefully considered. 
This ecological perspective motivates a systems-based approach for interrogating

2 and mapping the interactions between microbes and cancer or immune cells, similar to how

3 systems biologists have sought to systematically map protein-protein interactions and gene

4 knockout effects on cell phenotypes. Understanding the manipulable nodes and edges in

5 this multi-species network (including the host) can then guide rational interventions

6

7 directed towards a specific goal (e.g., cancer eradication). For example, researchers are investigating how modulation of the estrobolome, or the aggregate of enteric bacteria that metabolize estrogen, can influence hormonal or metabolic pathways that promote tumorigenesis [I09]. Microbial species with $\beta$-glucuronidase and $\beta$-glucuronide activities are manipulable 'nodes' in this network that facilitate estrogen metabolism and secretion. Targeting these microbial 'nodes' can modify the network so as to reduce the emergence of hormone-dependent cancers (Type I (endometrioid) endometrial cancer, estrogen receptorpositive breast cancer, and some ovarian cancers) [6o]. However, what is needed to enable this form of systems medicine are precision tools that alter individual or groups of microbes rather than whole communities, which is currently limited by most antibiotics, prebiotics, postbiotics, dietary interventions, and fecal microbiota transplants [34,5I,IIO]. Nonetheless, as the microbiota expands the cancer therapeutic armamentarium, oncologists are no longer simply manipulating or targeting host-centric, tumor-intrinsic properties.

\section{Conclusions and future perspectives}

This review aimed to elucidate the historical, conceptual, and scientific implications of recent data on the cancer-associated microbiota for oncology. We started with the notion that the microbiota comprises not simply another environmental factor among others that influences cancer growth and treatment but is intimately bound up with it. We then evaluated the relevance of this intimacy for oncology and explored its implications for establishing novel biomarkers, determining causality, and manipulating the microbiota to enhance or hinder treatments (see Outstanding Questions). We strove to capture the distinct conceptual aspects and therapeutic implications of this on-going research (Figure 2) while acknowledging its limitations. 


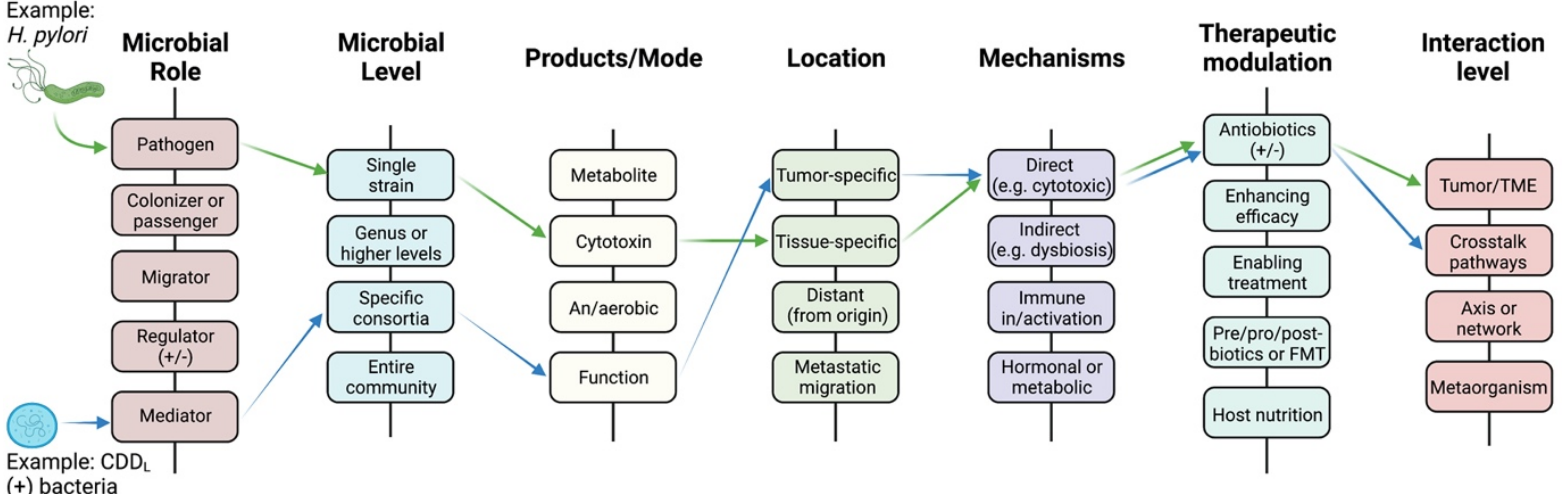

2

3

4 Going forward, we encourage further refinement of the proposals that articulate 5 microbiota-cancer-host 'axes' [I8,92,III], 'networks' [72,86], and 'systems' [88-9I]. Clarifying 6 the precise components of these networks and how they interact holds promise to help 7 contextualize novel biomarkers, to specify other causal mechanisms, and to rationally guide 8 therapeutic interventions that focus on the most effective targets.

9

10

11 


\section{Acknowledgements and Funding}

2 T.P. and J.S. have received funding from the European Research Council (ERC) under the

3 European Union's Horizon 2020 research and innovation programme - grant agreement $\mathrm{n}^{\circ}$

4637647 - IDEM (P.I.: T. Pradeu). After the completion of the IDEM project, J.S. was funded

5 by the University of Bordeaux, Région Nouvelle-Aquitaine and SIRIC BRIO. G.D.S.-P. is

6 funded by a fellowship from the National Cancer Institute within the National Institutes of

7 Health (F30 CA243480). R.K. is funded in part by grants from the National Cancer Institute

8 within the National Institutes of Health (RoI CA255206 U24 CA248454).

9

10

11 Disclosures

12 G.D.S.-P. and R.K. are inventors on a US patent application (PCT/US2OI9/059647) submitted 13 by The Regents of the University of California and licensed by Micronoma; that application 14 covers methods of diagnosing and treating cancer using microbial biomarkers in blood and 15 cancer tissues. G.D.S.-P. and R.K. are founders of and report stock interest in Micronoma. 16 G.D.S.-P. has filed several additional US patent applications on cancer microbiome diagnostics that are owned by The Regents of the University of California. R.K. additionally is a member of the scientific advisory board for GenCirq, holds an equity interest in GenCirq, and can receive reimbursements for expenses up to US \$5,000 per year. 


\section{References}

1 Solary, E. and Laplane, L. (2020) The role of host environment in cancer evolution. Evol Appl 13, 1756-1770

2 Nejman, D. et al. (2020) The human tumor microbiome is composed of tumor typespecific intracellular bacteria. Science 368, 973-980

3 Poore, G.D. et al. (2020) Microbiome analyses of blood and tissues suggest cancer diagnostic approach. Nature 579, 567-574

4 Tang, K.-W. et al. (2013) The landscape of viral expression and host gene fusion and adaptation in human cancer. Nat Commun 4, 2513

5 Aykut, B. et al. (2019) The fungal mycobiome promotes pancreatic oncogenesis via activation of MBL. Nature 574, 264-267

6 Zitvogel, L. et al. (2018) The microbiome in cancer immunotherapy: Diagnostic tools and therapeutic strategies. Science 359, 1366-1370

7 Clemente, J.C. et al. (2012) The Impact of the Gut Microbiota on Human Health: An Integrative View. Cell 148, 1258-1270

8 Gilbert, S.F. et al. (2012) A Symbiotic View of Life: We Have Never Been Individuals. The Quarterly Review of Biology 87, 325-341

9 McFall-Ngai, M. et al. (2013) Animals in a bacterial world, a new imperative for the life sciences. Proc Natl Acad Sci USA 110, 3229-3236

10 Gilbert, J.A. et al. (2018) Current understanding of the human microbiome. Nat Med 24, 392-400

11 Dobosz, P. and Dzieciątkowski, T. (2019) The Intriguing History of Cancer Immunotherapy. Front. Immunol. 10, 2965

12 Kadosh, E. et al. (2020) The gut microbiome switches mutant p53 from tumoursuppressive to oncogenic. Nature 586, 133-138

13 Ajami, N.J. and Wargo, J.A. (2020) Al finds microbial signatures in tumours and blood across cancer types. Nature 579, 502-503

14 Geller, L.T. et al. (2017) Potential role of intratumor bacteria in mediating tumor resistance to the chemotherapeutic drug gemcitabine. Science 357, 1156-1160

15 Riquelme, E. et al. (2019) Tumor Microbiome Diversity and Composition Influence Pancreatic Cancer Outcomes. Cell 178, 795-806.e12

16 Wong-Rolle, A. et al. (2020) Unexpected guests in the tumor microenvironment: microbiome in cancer. Protein Cell DOI: 10.1007/s13238-020-00813-8

17 Lou, X. et al. (2021) Bacteria-Mediated Synergistic Cancer Therapy: Small Microbiome Has a Big Hope. Nano-Micro Lett. 13, 37

18 Sepich-Poore, G.D. et al. (2021) The microbiome and human cancer. Science 371, eabc4552

19 Singh, R.P. et al. (2021) Emerging role of microbiota in immunomodulation and cancer immunotherapy. Seminars in Cancer Biology 70, 37-52

20 Oiseth, S.J. and Aziz, M.S. (2017) Cancer immunotherapy: a brief review of the history, possibilities, and challenges ahead. JCMT 3, 250

21 Hoption Cann, S.A. et al. (2003) Dr William Coley and tumour regression: a place in history or in the future. Postgrad Med J 79, 672

22 Starnes, C.O. (1992) Coley's toxins in perspective. Nature 357, 11-12

23 White, M.K. et al. (2014) Viruses and Human Cancers: a Long Road of Discovery of Molecular Paradigms. Clin Microbiol Rev 27, 463-481 
24 ACS (1990) Unproven Methods in Cancer Management: Livingston-Wheeler therapy. CA: A Cancer Journal for Clinicians 40, 103-108

25 Glover, T.J. (1930) The bacteriology of cancer. Canada Lancet and Practitioner 75, 92111

26 Seibert, F.B. et al. (1972) Bacteria in tumors. Transactions of the New York Academy of Sciences 34, 504-533

27 Rous, P. (1911) A sarcoma of the fowl transmissible by an agent separable from the tumor cells. Journal of Experimental Medicine 13, 397-411

28 Sankaran, N. (2014) When viruses were not in style: Parallels in the histories of chicken sarcoma viruses and bacteriophages. Studies in History and Philosophy of Science Part C: Studies in History and Philosophy of Biological and Biomedical Sciences 48, 189-199

29 The Nobel Assembly at the Karolinska Institute (1989) Nobel Press Release 1989,

30 Weinberg, R.A. (1998) Racing to the beginning of the road: the search for the origin of cancer, W.H. Freeman.

31 Gopalakrishnan, V. et al. (2018) Gut microbiome modulates response to anti-PD-1 immunotherapy in melanoma patients. Science 359, 97-103

32 Viaud, S. et al. (2013) The Intestinal Microbiota Modulates the Anticancer Immune Effects of Cyclophosphamide. Science 342, 971-976

33 Elinav, E. et al. (2013) Inflammation-induced cancer: crosstalk between tumours, immune cells and microorganisms. Nat Rev Cancer 13, 759-771

34 McQuade, J.L. et al. (2019) Modulating the microbiome to improve therapeutic response in cancer. The Lancet Oncology 20:e77-e91,

35 Buchta Rosean, C. et al. (2019) Preexisting Commensal Dysbiosis Is a Host-Intrinsic Regulator of Tissue Inflammation and Tumor Cell Dissemination in Hormone ReceptorPositive Breast Cancer. Cancer Res 79, 3662-3675

36 Behrouzi, A. et al. (2019) The significance of microbiome in personalized medicine. Clinical and Translational Medicine 8,

37 FDA-NIH Biomarker Working Group (2016) BEST (Biomarkers, EndpointS, and other Tools) Resource, Food and Drug Administration (US).

38 Kwong, T.N.Y. et al. (2018) Association Between Bacteremia From Specific Microbes and Subsequent Diagnosis of Colorectal Cancer. Gastroenterology 155, 383-390.e8

39 Kashyap, P.C. et al. (2017) Microbiome at the Frontier of Personalized Medicine. Mayo Clinic Proceedings 92, 1855-1864

40 Huybrechts, I. et al. (2020) The Human Microbiome in Relation to Cancer Risk: A Systematic Review of Epidemiologic Studies. Cancer Epidemiol Biomarkers Prev 29, 1856-1868

41 Tsay, J.-C.J. et al. (2020) Lower airway dysbiosis affects lung cancer progression. Cancer Discov DOI: 10.1158/2159-8290.CD-20-0263

42 Zhou, Z. et al. (2020) Human Gut Microbiome-Based Knowledgebase as a Biomarker Screening Tool to Improve the Predicted Probability for Colorectal Cancer. Front. Microbiol. 11, 596027

43 Zhang, C. et al. (2020) The Potential Gut Microbiota-Mediated Treatment Options for Liver Cancer. Front. Oncol. 10, 524205

44 Fan, X. et al. (2018) Human oral microbiome and prospective risk for pancreatic cancer: a population-based nested case-control study. Gut 67, 120-127

45 Zhou, C.-B. et al. (2021) Gut Microbiota in Cancer Immune Response and Immunotherapy. Trends in Cancer 7, 647-660 
46 Pushalkar, S. et al. (2018) The Pancreatic Cancer Microbiome Promotes Oncogenesis by Induction of Innate and Adaptive Immune Suppression. Cancer Discov 8, 403-416

47 Pinato, D.J. et al. (2019) Association of Prior Antibiotic Treatment With Survival and Response to Immune Checkpoint Inhibitor Therapy in Patients With Cancer. JAMA Oncol 5,1774

48 Dzutsev, A. et al. (2017) Microbes and Cancer. Annual Review of Immunology 35, 199228

49 Ribas, A. and Wolchok, J.D. (2018) Cancer immunotherapy using checkpoint blockade. Science 359, 1350-1355

50 Daillère, R. et al. (2016) Enterococcus hirae and Barnesiella intestinihominis Facilitate Cyclophosphamide-Induced Therapeutic Immunomodulatory Effects. Immunity 45, 931943

51 Baruch, E.N. et al. (2020) Fecal microbiota transplant promotes response in immunotherapy-refractory melanoma patients. Science DOI: 10.1126/science.abb5920

52 Davar, D. et al. (2021) Fecal microbiota transplant overcomes resistance to anti-PD-1 therapy in melanoma patients. Science 371, 595-602

53 Finlay, B.B. et al. (2020) Can we harness the microbiota to enhance the efficacy of cancer immunotherapy? Nature Reviews Immunology DOI: 10.1038/s41577-020-0374-6

54 Shahanavaj, K. et al. (2015) Cancer and the microbiome: potential applications as new tumor biomarker. Expert Review of Anticancer Therapy 15, 317-330

55 Scott, A.J. et al. (2019) International Cancer Microbiome Consortium consensus statement on the role of the human microbiome in carcinogenesis. Gut 68, 1624-1632

56 IARC Working Group (2012) Biological agents. Volume 100 B. A review of human carcinogens. IARC Monogr Eval Carcinog Risks Hum 100, 1-441

57 de Martel, C. et al. (2020) Global burden of cancer attributable to infections in 2018: a worldwide incidence analysis. The Lancet Global Health 8, e180-e190

58 Dzutsev, A. et al. (2015) The role of the microbiota in inflammation, carcinogenesis, and cancer therapy. Eur J Immunol 45, 17-31

59 Janney, A. et al. (2020) Host-microbiota maladaptation in colorectal cancer. Nature 585, 509-517

60 Plottel, C.S. and Blaser, M.J. (2011) Microbiome and Malignancy. Cell Host \& Microbe 10, 324-335

61 Bullman, S. et al. (2017) Analysis of Fusobacterium persistence and antibiotic response in colorectal cancer. Science 358, 1443-1448

62 Ainsworth, C. (2017) Microbiome: A bag of surprises. Nature 551, S40-S41

63 Bertocchi, A. et al. (2021) Gut vascular barrier impairment leads to intestinal bacteria dissemination and colorectal cancer metastasis to liver. Cancer Cell 39, 708-724.e11

64 Mantovani, A. et al. (2008) Cancer-related inflammation. Nature 454, 436-444

65 Trinchieri, G. (2015) Cancer Immunity: Lessons From Infectious Diseases. J Infect Dis 212:S67-S73,

66 Petersen, C. and Round, J.L. (2014) Defining dysbiosis and its influence on host immunity and disease. Cell Microbiol 16, 1024-1033

67 Olesen, S.W. and Alm, E.J. (2016) Dysbiosis is not an answer. Nat Microbiol 1, 16228

68 Hooks, K.B. and O'Malley, M.A. (2017) Dysbiosis and Its Discontents. mBio 8, e01492-17

69 Meisel, M. et al. (2018) Microbial signals drive pre-leukaemic myeloproliferation in a Tet2-deficient host. Nature 557, 580-584 
70 Routy, B. et al. (2018) The gut microbiota influences anticancer immunosurveillance and general health. Nat Rev Clin Oncol 15, 382-396

71 Routy, B. et al. (2018) Gut microbiome influences efficacy of PD-1-based immunotherapy against epithelial tumors. Science 359, 91-97

72 Xavier, J.B. et al. (2020) The Cancer Microbiome: Distinguishing Direct and Indirect Effects Requires a Systemic View. Trends in Cancer 6, 192-204

73 Barrett, M. et al. (2020) Mutagenesis by Microbe: the Role of the Microbiota in Shaping the Cancer Genome. Trends in Cancer 6, 277-287

74 Silva-García, O. et al. (2019) Wnt/ß-Catenin Signaling as a Molecular Target by Pathogenic Bacteria. Front. Immunol. 10, 2135

75 Wilson, M.R. et al. (2019) The human gut bacterial genotoxin colibactin alkylates DNA. Science 363 , eaar7785

$76 \mathrm{Ma}$, C. et al. (2018) Gut microbiome-mediated bile acid metabolism regulates liver cancer via NKT cells. Science 360,

77 Jin, C. et al. (2019) Commensal Microbiota Promote Lung Cancer Development via $ү \delta \mathrm{T}$ Cells. Cell 176, 998-1013.e16

78 Parhi, L. et al. (2020) Breast cancer colonization by Fusobacterium nucleatum accelerates tumor growth and metastatic progression. Nat Commun 11, 3259

79 Chen, J. et al. (2019) Effects of Intestinal Microbial-Elaborated Butyrate on Oncogenic Signaling Pathways. Nutrients 11, 1026

80 Garrett, W.S. (2015) Cancer and the microbiota. Science 348, 80-86

81 Zagato, E. et al. (2020) Endogenous murine microbiota member Faecalibaculum rodentium and its human homologue protect from intestinal tumour growth. Nat Microbiol 5, 511-524

82 Bessell, C.A. et al. (2020) Commensal bacteria stimulate antitumor responses via T cell cross-reactivity. JCl Insight 5,

83 Fluckiger, A. et al. (2020) Cross-reactivity between tumor MHC class I-restricted antigens and an enterococcal bacteriophage. Science 369, 936-942

84 Walter, J. et al. (2020) Establishing or Exaggerating Causality for the Gut Microbiome: Lessons from Human Microbiota-Associated Rodents. Cell 180, 221-232

85 Elinav, E. et al. (2019) The cancer microbiome. Nat Rev Cancer 19, 371-376

86 Gentles, A.J. and Gallahan, D. (2011) Systems biology: confronting the complexity of cancer. Cancer Res 71, 5961-5964

87 Werner, H.M.J. et al. (2014) Cancer Systems Biology: a peek into the future of patient care? Nat Rev Clin Oncol 11, 167-176

$88 \mathrm{Du}, \mathrm{W}$. and Elemento, O. (2015) Cancer systems biology: embracing complexity to develop better anticancer therapeutic strategies. Oncogene 34, 3215-3225

89 Archer, T.C. et al. (2016) Systems Approaches to Cancer Biology. Cancer Res 76, 67746777

90 Plutynski, A. and Bertolaso, M. (2018) What and How Do Cancer Systems Biologists Explain? Philosophy of Science, juillet DOI: 10.1086/699716.

91 Reticker-Flynn, N.E. and Engleman, E.G. (2020) Cancer systems immunology. Elife 9,

92 Sharma, P. et al. (2020) Gut Microbiome: The Third Musketeer in the Cancer-Immune System Cross-Talk. Journal of Pancreatology 3, 181-187

93 Lemon, K.P. et al. (2012) Microbiota-Targeted Therapies: An Ecological Perspective. Science Translational Medicine 4, 137rv5-137rv5 
94 Martins Lopes, M.S. et al. (2020) Antibiotics, cancer risk and oncologic treatment efficacy: a practical review of the literature. Ecancermedicalscience 14, 1106

95 Kroemer, G. and Zitvogel, L. (2018) Cancer immunotherapy in 2017: The breakthrough of the microbiota. Nature Reviews Immunology 18, 87-88

96 Matson, V. et al. (2018) The commensal microbiome is associated with anti-PD-1 efficacy in metastatic melanoma patients. Science 359, 104-108

97 Vétizou, M. et al. (2015) Anticancer immunotherapy by CTLA-4 blockade relies on the gut microbiota. Science 350, 1079-1084

98 Mager, L.F. et al. (2020) Microbiome-derived inosine modulates response to checkpoint inhibitor immunotherapy. Science 369, 1481-1489

99 Griffin, M.E. et al. (2021) Enterococcus peptidoglycan remodeling promotes checkpoint inhibitor cancer immunotherapy. Science 373, 1040-1046

100 Roy, S. and Trinchieri, G. (2017) Microbiota: a key orchestrator of cancer therapy. Nat Rev Cancer 17, 271-285

101 Ansaldo, E. and Belkaid, Y. (2021) How microbiota improve immunotherapy. Science $373,966-967$

102 Guo, H. et al. (2020) Multi-omics analyses of radiation survivors identify radioprotective microbes and metabolites. Science 370, eaay9097

103 Shiao, S.L. et al. (2021) Commensal bacteria and fungi differentially regulate tumor responses to radiation therapy. Cancer Cell DOI: 10.1016/j.ccell.2021.07.002

104 lida, N. et al. (2013) Commensal Bacteria Control Cancer Response to Therapy by Modulating the Tumor Microenvironment. Science 342, 967-970

105 Schwabe, R.F. and Jobin, C. (2013) The microbiome and cancer. Nat Rev Cancer 13, 800-812

106 Sedighi, M. et al. (2019) Therapeutic bacteria to combat cancer; current advances, challenges, and opportunities. Cancer Med DOI: 10.1002/cam4.2148

107 Brown, C. (2020) Scientists are harnessing viruses to treat tumours. Nature 587, S60$\mathrm{S} 62$

108 Barton, E.S. et al. (2007) Herpesvirus latency confers symbiotic protection from bacterial infection. Nature 447, 326-329

109 Komorowski, A.S. and Pezo, R.C. (2020) Untapped "-omics": the microbial metagenome, estrobolome, and their influence on the development of breast cancer and response to treatment. Breast Cancer Res Treat 179, 287-300

$110 \mathrm{Li}$, Y. et al. (2020) Prebiotic-Induced Anti-tumor Immunity Attenuates Tumor Growth. Cell Reports 30, 1753-1766.e6

111 Jain, T. et al. (2021) New Insights Into the Cancer-Microbiome-Immune Axis: Decrypting a Decade of Discoveries. Front. Immunol. 12, 622064 
3 Figure I. Microbiota-tumor-host interactions. Visual depiction of some key mechanisms

4 and causal pathways being uncovered within the host-tumor-microbiota network.

5

6

Figure 2. Microbiota-Cancer Conceptual Matrix. This matrix, depicted as a parallel coordinates plot, reflects various conceptual and empirical issues in current microbiotacancer research while engendering novel questions. For instance, research has shown how the microbiota can play different roles in tumorigenesis and treatment, but it remains an open question whether these roles are based on a particular microbial species, function, or anatomical location, and the precise mechanisms involved are often tentative due to the complexity of the cancer-host interactions. It further remains important to elucidate how these roles and levels intersect with immune-microbiota pathways and whether they are conserved amongst multiple microbes in a particular environment. Next, we can modulate treatments through the targeted use of antibiotics or host nutritional interventions and explore their efficacy in altering not only tumor- or tissue-specific microbiota, but also preventing microbial translocation and the formation of premetastatic niches. Finally, the complexity of causal mechanisms and therapeutic interventions increases as we shift analysis from the TME level to interacting networks or even the entire metaorganism. 